\title{
Inverse Modeling for Large-Eddy Simulation
}

\author{
Bernard J. Geurts \\ Faculty of Applied Mathematics, J.M. Burgers Center, University of Twente \\ P.O. Box 217, 7500 AE Enschede, The Netherlands
}

\begin{abstract}
Approximate higher order polynomial inversion of the top-hat filter is developed with which the turbulent stress tensor in Large-Eddy Simulation can be consistently represented using the filtered field. Generalized (mixed) similarity models are proposed which improved the agreement with the kinetic energy transfer to small scales. These similarity models are analyzed for random periodic signals and the ensemble averaged spectra of the turbulent stress tensor and the corresponding models are compared.
\end{abstract}

\section{INTRODUCTION}

The numerical simulation of transitional and turbulent flows forms a field of considerable interest. Direct numerical simulation (DNS) of such flows in simple geometries is nowadays possible, but requires a full spatial and temporal resolution of all relevant scales of motion. For more complex flows modeling of the equations, which reduces the degrees of freedom of the dynamical system, is required. A concise modeling arises in large-eddy simulation (LES) and involves a spatial convolution filter [1-3]. Filtering the nonlinear terms in the Navier-Stokes equations leads to contributions which contain the unfiltered solution and require modelling. The filter appears explicitly in the turbulent stress tensor for which various models have been proposed, some of which are formulated independent of a specific filter. Moreover, the application of two different filters does not alter the mathematical structure of the resulting filtered equations. This 'independence' of the adopted filter is quite undesirable if a detailed comparison between DNS and LES is attempted e.g. to assess the quality of 'subgrid-models' or to separate modeling-errors from numerical errors [4]. Disregarding numerical errors, a 'perfect' LES would require the DNS solution and the selection of a specific filter. Then, the filtered nonlinear terms can be evaluated exactly at each instant leading to $\overline{u_{\mathrm{DNS}}}=u_{\mathrm{LES}}$ where $u_{\text {DNS }}\left(u_{\text {LES }}\right)$ denotes the DNS (LES) solution and the bar represents the filter.

From this observation one may anticipate that if $u_{\mathrm{DNS}}$ can be recovered approximately to some degree from $u_{\text {LES }}$ through an appropriate 'inversion' of the filteroperation, a suitable subgrid-model may be obtained for LES. This approach involves the specific filter explicitly and leads to generalized similarity models. Recovering $u_{\text {DNS }}$ from $u_{\text {LES }}$ can be realized accurately for solutioncomponents with wave-lengths up to the filter-width. Contributions from yet smaller scale components can not be recovered accurately and additional physical information is required to model the full turbulent stress tensor [5]. In this paper we develop approximate higher order polynomial inversion for the top-hat filter in section II and study the resulting generalized similarity models in section III for random periodic solutions in 1D as well as for $3 \mathrm{D}$ turbulent flow in a mixing layer [3]. In section IV we summarize our findings.

\section{HIGHER ORDER POLYNOMIAL INVERSION}

In this section we construct approximate higher order polynomial inversion with which the original solution can be recovered to some degree from the filtered solution. We adopt the top-hat filter in real space as 'basic' filter although the approach can also be developed for other (higher order) compact support filters [6].

The filtering of a signal $u$ is defined by $u \rightarrow \bar{u}$ :

$$
\bar{u}(x)=\int_{x-\Delta / 2}^{x+\Delta / 2} \frac{u(\xi)}{\Delta} d \xi
$$

where $\Delta$ denotes the filter-width. The filter may also be written as a convolution integral and the Fouriertransform of the filter-kernel $H=\sin (k \Delta / 2) /(k \Delta / 2)$. It can be shown that $H(k \Delta)-1 \sim(k \Delta)^{2}$ if $(k \Delta)$ is small and an effective damping occurs if $k \Delta$ becomes large.

The Fourier-transform $H$ has infinitely many roots which implies that the filter-operation has no exact inverse. To reconstruct the signal $u$ from $\bar{u}$ we introduce approximate inversion. This is based on the requirement that polynomials up to a certain degree are invariant under the combined action of the filter and its approximate inverse. For this purpose we introduce the inversionkernel $\mathcal{J}_{L}(y)=(1 / \Delta) \sum_{m=0}^{L} a_{2 m}^{(L)} y^{2 m}$ with $y=(x-\xi) / \Delta$. The coefficients $\left\{a_{2 m}^{(L)}\right\}$ are constructed such that

$$
\int_{x-\Delta / 2}^{x+\Delta / 2} d \xi \mathcal{J}_{L}((x-\xi) / \Delta) \int_{\xi-\Delta / 2}^{\xi+\Delta / 2} d s \frac{s^{2 m}}{\Delta}=x^{2 m}
$$

for $m=0, . ., L$ which implies a linear system of equations for $\left\{a_{2 m}^{(L)}\right\}$. The inversion is denoted by the hatsymbol and implies $\widehat{x^{m}}=x^{m}$ for all $m=0, . ., 2 L+1$. In table I some inversion operators are listed.

TABLE I. Inversion-kernels $\Delta \mathcal{J}_{L}$ for the top-hat filter.

\begin{tabular}{ll}
\hline \hline$L$ & $\Delta \mathcal{J}_{L}$ \\
0 & 1 \\
1 & $-30 y^{2}+\frac{7}{2}$ \\
2 & $2310 y^{4}-525 y^{2}+\frac{127}{8}$ \\
3 & $-396396 y^{6}+137445 y^{4}-\frac{47145}{4} y^{2}+\frac{2399}{16}$ \\
\hline \hline
\end{tabular}


One may show that $\tau \sim m_{L} \sim \Delta^{2}$. These models

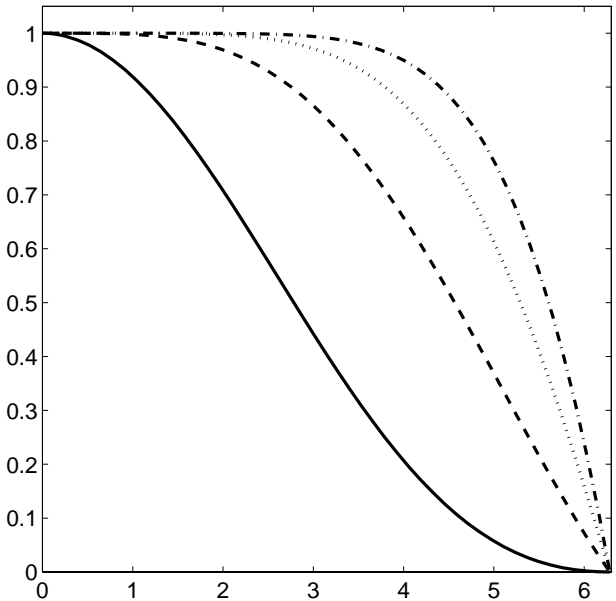

FIG. 1. Fourier-transform $G_{L}$ versus $k \Delta$ of the top-hat filter combined with $\mathcal{J}_{L}$ for $L=0$ (solid), $L=1$ (dashed), $L=2$ (dotted) and $L=3$ (dash-dotted).

In order to quantify the effectiveness of the approximate inversion we consider the application of the top-hat filter to $u=\sin (k x)$ followed by the application of an approximate inversion. Since the approximate inverse can be written as a convolution integral it is sufficient to consider the Fourier-transform $G_{L}$ of the combined operation since $\widehat{\bar{u}}=G_{L} u$. In figure 1 we plotted $G_{L}$ for increasing inversion-order. If $k \Delta$ is small it may be shown that $G_{L}=1-\mathcal{O}\left((k \Delta)^{2(L+1)}\right)$. One clearly observes a significant improvement in the reconstructed signal if $L$ is increased since $G_{L}$ is much closer to 1 over a wider range of $k \Delta$. In particular, an almost perfect recovery is accomplished for modes with $k \Delta$ up to $\approx \pi$ if $L \geq 2$. In combination with the Fourier-transform $H$ of the tophat filter, we also infer that the inversion operation assumes large values as $k \Delta$ approaches $2 \pi$, i.e. small scale solution-components (and numerical errors) in $u_{\text {LES }}$ will be greatly amplified by the approximate inversion if $L$ is large. These contributions require special attention and may necessitate the introduction of additional physical modeling.

\section{GENERALIZED SIMILARITY MODELS}

In this section we formulate generalized similarity models and compare them with the stress tensor $\tau=$ $\overline{u^{2}}-\bar{u}^{2}$ if $u$ is a random periodic signal in one dimension. Subsequently we study the dissipative character of these models using DNS-data obtained from turbulent flow in a temporal mixing layer [3].

Consistent with the application of the filter in combination with a $2(L+1)$-th order approximate inversion we introduce the following class of models:

$$
m_{L}={\overline{\widehat{\bar{u}}^{2}}}^{2} \overline{\overline{\mathrm{u}}}^{2}
$$

form a generalization of Bardina's similarity model [5] $m_{B}=\overline{\bar{u}}^{2}-\overline{\bar{u}}^{2}$ which arises if the inversion operator is the identity. This model is known to have a high correlation with $\tau$, but does not adequately describe the dissipation of the small scales and overestimates the backscatter [3]. As will be shown, an increase in $L$ leads to more accurate models for $\tau$ which may resolve these shortcomings.

The properties of $m_{L}$ may be illustrated with a Fourieranalysis. We first consider $u=\sin (k x)$ for which

$$
\tau=\frac{1}{2}\left[1-H^{2}(k \Delta)\right]-\frac{1}{2}\left[H(2 k \Delta)-H^{2}(k \Delta)\right] \cos (2 k x)
$$

and $m_{L}=G_{L}^{2}(k \Delta) \tau$ i.e. an accurate model if $G_{L}(k \Delta) \approx$ 1. In figure 2 we show the amplitude $(A)$ of the oscillating contribution to $\tau$ and $m_{L}$ which demonstrates the effectiveness of the reconstruction as $L$ increases.

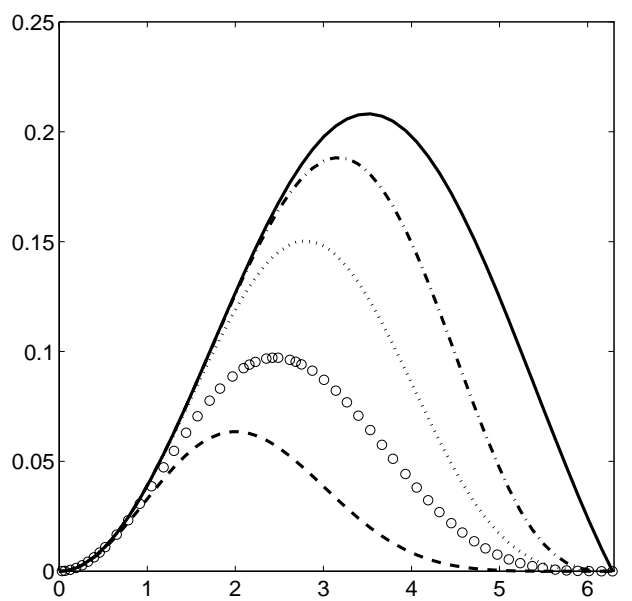

FIG. 2. Amplitude $A$ corresponding to $\tau$ (solid) and the models $m_{L}$ for $L=0$ (dashed), $L=1$ (dotted) and $L=2$ (dash-dotted) and Bardina (o).

This single mode analysis can be extended to general random periodic signals $u=\sum c_{k} e^{i k x}$. Signals consistent with a spectrum $\mathcal{E}$ can be obtained if $c_{k}=$ $\sqrt{\mathcal{E}(k) / 2} \exp \left(i \theta_{k}\right)$ where $\left\{\theta_{k}\right\}$ are uniformly distributed stochastic variables on $[-\pi, \pi$ [. This yields for $\tau$ :

$$
\tau=\sum_{n=-\infty}^{\infty} d_{n} e^{i n x} ; \quad d_{n}=\sum_{k=-\infty}^{\infty} c_{k} c_{n-k} F_{n, k}
$$

where $F_{n, k}=[H(n \Delta)-H(k \Delta) H((n-k) \Delta)]$ are 'reduction factors'. For $m_{L}$ we find a similar result with reduction factors $T_{n, k}=G_{L}(k \Delta) G_{L}((n-k) \Delta) F_{n, k}$. A specific realization of $u$ has a corresponding spectrum $D(n)=\left|d_{n}\right|^{2}+\left|d_{-n}\right|^{2}$ of $\tau$ which can be determined from (4). The ensemble averaged spectrum $\langle D(n)\rangle$ is given by

$$
\langle D(n)\rangle=\sum_{k=-\infty}^{\infty} \mathcal{E}(k) \mathcal{E}(n-k) F_{n, k}^{2}
$$






FIG. 3. Averaged spectra versus $n$ of $\tau$ (solid) and $m_{L}$ with $L=0$ (dashed), $L=1$ (dotted), $L=2$ (dash-dotted) and Bardina (o) with $\Delta=0.01$ and spectrum-parameters $m=4, k_{1}=10$ and $k_{2}=40$.

In figure 3 an example is shown in which

$$
\mathcal{E}(k)=k^{-5 / 3} \mathcal{M}\left(\left(k / k_{1}\right)^{m}\right) \mathcal{M}\left(k^{5 / 3} \exp \left(-\left(k / k_{2}\right)\right)\right)
$$

where $\mathcal{M}(z)=1-\exp (-z)$. This model-spectrum shows a behavior $\mathcal{E}(k) \sim k^{m}$ if $k$ is small, an inertial subrange in which $\mathcal{E}(k) \sim k^{-5 / 3}$ and an exponential tail for large $k$. The correspondence between $\tau$ and $m_{L}$ is very accurate if $L$ is large enough and $1 / \Delta$ is chosen in the inertial subrange. Other model-spectra have also been considered and give rise to similar results.

This Fourier-analysis involves the explicit analytical filtering and approximate inversion of smooth known signals. In practice a solution typically is defined on a certain grid with spacing $h$ and filtered numerically. The numerical and analytical filtering are very close to eachother for $k$-values up to $2 \pi / \Delta$ if $\Delta / h \gtrsim 4$ using Newton-Cotes integration [6]. In a priori tests of the generalized similarity models using DNS-data of turbulent flow in a temporal mixing layer [3] a high correlation of $m_{L}$ with $\tau$ in the turbulent regime was observed which increases with $L$. In order to quantify the dissipative character of $m_{L}$ we considered dynamic mixed models with $m_{L}$ as base model combined with a dynamic eddy-viscosity contribution. An increase in $L$ at constant $\Delta$ leads to a decrease in the eddy-viscosity, consistent with the improved recovery of the DNS solution. Conversely at constant $L$ a sufficiently large increase in $\Delta$ may lead to an eddyviscosity term which is larger than the similarity part, indicating an inaccurate reconstruction of the DNS solution. The transfer of energy to small scales appears better represented by the generalized similarity models if the inversion order increases, provided $1 / \Delta$ is in the inertial range. In case $\Delta$ is too large additional dynamic eddy-viscosity modeling is required in order to represent the flow of energy to the subgrid scales.
Generalized similarity models have been constructed based on approximate inversion of the top-hat filter. These (dynamic mixed) similarity models appear quite promising for large-eddy simulation in view of the high correlation combined with an accurate representation of the kinetic energy transfer. The approximate inversion adds only a small amount of numerical calculation compared with other proposals. In the formulation of ref. [1] a system of higher order partial differential equations needs to be solved simultaneously with the LES equations. The approach in [7] is based on a second order differential equation for $u$ with source term $\bar{u}$ which also increases the simulation time noticeably. Future research is devoted to nonuniform filters in which the filter-width $\Delta$ depends on the spatial coordinate. These filters add an additional term to the filtered equations since $\overline{\partial_{x} u} \neq \partial_{x} \bar{u}$ which a priori is of comparable order of magnitude as the turbulent stress tensor [6]. The use of the latter type of filters is, however, required in order to systematically develop LES for complex flows.

\section{ACKNOWLEDGEMENTS}

Part of this research was supported by the National Aerospace Laboratory (NLR). Stimulating and clarifying discussions with Dr. Kuerten of the University of Twente have been very helpful.

[1] S. Ghosal, P. Moin, 'The basic equations for the Large Eddy simulation of turbulent flows in complex geometry', J. Comp. Phys., 118, 24, (1995)

[2] A.W. Vreman, B.J. Geurts, J.G.M. Kuerten, 'On the formulation of the dynamic mixed subgrid-scale model', Phys. Fluids A, 6, 4057, (1994)

[3] A.W. Vreman, B.J. Geurts, J.G.M. Kuerten, 'Large-eddy simulation of the turbulent mixing layer', JFM, 339, 357, (1997)

[4] A.W. Vreman, B.J. Geurts, J.G.M. Kuerten, 'Comparison of numerical schemes in Large Eddy Simulation of the temporal mixing layer', Int. J. Num. Meth. in Fluids, 22, 297, (1996)

[5] J. Bardina, J.H. Ferziger, W.C. Reynolds, 'Improved turbulence models based on LES of homogeneous incompressible turbulent flows', Department of Mechanical Engineering, Report No. TF-19, Stanford, (1983)

[6] B.J. Geurts, A.W. Vreman, J.G.M. Kuerten and R. van Buuren, 'Noncommuting filters and dynamic modelling for les of turbulent compressible flow in 3D shear layers', Direct and Large-Eddy Simulation II, Eds: P.R. Voke, L. Kleiser and J.P. Chollet, To appear, Kluwer Academic Publishers, (1997)

[7] K.B. Shah, J.H. Ferziger, 'A new non-eddy viscosity subgrid-scale model and its application to channel flow', CTR Briefs, p. 73, (1996) 